\title{
Impact of telemedicine on health outcomes in children with medical complexity: an integrative review
}

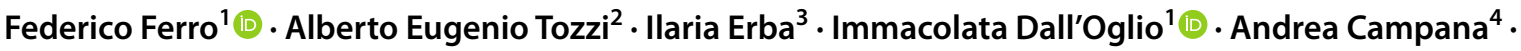 \\ Corrado Cecchetti ${ }^{5}$. Caterina Geremia ${ }^{4} \cdot$ Maria Luisa Rega ${ }^{6} \cdot$ Gloria Tontini $^{4} \cdot$ Emanuela Tiozzo $^{1}$. \\ Orsola Gawronski ${ }^{1}$ (i)
}

Received: 21 January 2021 / Revised: 10 June 2021 / Accepted: 12 June 2021 / Published online: 1 July 2021

(c) The Author(s), under exclusive licence to Springer-Verlag GmbH Germany, part of Springer Nature 2021

\begin{abstract}
Children with medical complexity (CMC) are a high priority population with chronic illnesses dependent on the use of health services, on technological systems to support their vital functions and characterized by multiple health needs. One of the main challenges linked to chronic conditions is finding solutions to monitor CMC at home, avoiding re-hospitalization and the onset of complications. Telemedicine enables to remotely follow up patients and families. An integrative review was performed to assess whether telemedicine improves health outcomes for CMC. Medline/PubMed, CINAHL, Cochrane Library, Web of Science, and Scopus were searched to identify studies describing the effect of using telemedicine systems on health outcomes for CMC. The PRISMA guidelines were used to select the papers. The methodological quality of the studies was evaluated through the Johanna Briggs Institute critical appraisal tools and the Cochrane Collaboration ROB 2.0. A total of 17 papers met the quality criteria and were included. Specialized telemedicine systems (tele-visits), telehealth, and tele-monitoring have been reported to reduce unplanned hospitalizations and visits, decrease total costs for healthcare services and families, and increase satisfaction for family members. No effect was found on the quality of life in children and their families.

Conclusion: Available evidence supporting the use of telemedicine in CMC is favorable but limited. High-quality methodological studies including other unexplored health outcomes such as mental health, hospital readmissions, mortality, caregiver competences, and self-efficacy are needed to confirm the effectiveness of telemedicine systems in improving health outcomes for CMC.

\section{What is Known:}

- CMC are an extremely fragile patient population with frequent access to healthcare services compared with other chronic conditions.

- There is conflicting evidence of the effectiveness of telemedicine clinical outcomes, healthcare utilization, and costs in pediatrics.

What is New:

- There is some evidence that for CMC, telemedicine reduces unplanned hospitalizations, healthcare service costs, and financial burden for

families, while increasing caregivers' satisfaction with care.

- Further research is needed to confirm the effectiveness of telemedicine systems in improving health for CMC.
\end{abstract}

Keywords Children Chronic diseases $\cdot$ Medical complexity $\cdot$ Special needs $\cdot$ Telemedicine $\cdot$ Telehealth $\cdot$ Health outcomes

$\begin{array}{ll}\text { Abbreviations } \\ \text { APRN } & \text { Advanced Practice Registered Nurse } \\ \text { CINAHL } & \text { Cumulative Index of Nursing and Allied } \\ & \text { Health Literature }\end{array}$

Communicated by Gregorio Paolo Milani

Orsola Gawronski

orsola.gawronski@opbg.net
CG-CAHPS Consumer Assessment of Healthcare Providers and Systems Clinician and Group survey

CMC Children with medical complexity

Covid-19 Coronavirus 2019

CSHCN Children with special health-care needs

ED Emergency Department

FCC Family centered care

HRQOL Health-related quality of life

Extended author information available on the last page of the article 


$\begin{array}{ll}\text { PICO } & \begin{array}{l}\text { Population, intervention, comparison and } \\ \text { outcome } \\ \text { Preferred Reporting Items for Systematic }\end{array} \\ \text { PRISMA } & \begin{array}{l}\text { Revies and Meta-Analyses } \\ \text { Randomized controlled trial }\end{array} \\ \text { RCT } & \begin{array}{l}\text { Telemedicine } \\ \text { TM }\end{array} \\ \text { UHR } & \text { Unplanned hospital readmissions }\end{array}$

\section{Introduction}

Hospitalizations of children with chronic diseases with special health-care needs (CSHCN) are increasing, particularly in children with significant chronic conditions because of higher survival rates resulting from more effective medical treatments $[1,2]$. Children with medical complexity (CMC), a subcategory of CSHCN, are defined as children with known chronic conditions often associated with medical fragility, special needs that affect the use of healthcare services and families' financial resources, serious functional limitations often associated with technology dependence, and the need for multi-professional interventions [3]. It is estimated that $\mathrm{CMC}$ are $<1 \%$ of the total pediatric population in Canada and the USA [4, 5]. However, CMC are at high risk of hospitalization after admission to the Emergency Department (ED) (19-25\%), re-hospitalizations after discharge, and increase healthcare expenditure [2, 6-9] [10].

Medical complexities in these children often lead to the development of complications, depending on the child's disease [3] involving frequent access to healthcare services, continuous home care, and remote education and support for their families. Moreover, families of CMC living far from large urban centers and specialized clinics may have limited access to treatment, also due to travelling costs $[11,12]$.

Telemedicine (TM) has been widely used in healthcare for patients with chronic conditions needing frequent follow-up visits and medical interventions [13]. TM has been applied in CMC using primary care models, consultative or co-management models, and episode-based models [14]. Primary care models-like the Medical Home Model [15-20] — use TM to create a bridge between hospitals and the community. Specialized center-based telemedicine is currently used to prevent CMC clinical deterioration and promote wellbeing at home, support families, and homecare teams and care-coordination [21, 22].

Several studies have been conducted to assess the impact of TM in chronic patients, including type I diabetes [23-27], asthma [28-30], obesity [27, 31, 32], cystic fibrosis [33-35], mental disorders [36], autism [37-39], and chronic pain [40]. Several TM interventions on pediatric populations also target their families, to increase coordination of care, quality of life, and reduce the financial burden [31, 38]. These studies, however, provide inconsistent evidence on clinical outcomes, healthcare utilization and costs [41]. CMC could benefit from TM also to reduce the risk of exposure to viral infections such as the coronavirus disease 2019 avoiding standard hospital and outpatient care [42].

To our knowledge, there are no systematic reviews on the effectiveness of TM on CMC health outcomes, costs, and healthcare utilization. Therefore, we performed an integrative review to study the association of telemedicine with family and child well-being, child health, family's ability to engage in self-care, adaptive functioning, satisfaction with $\mathrm{TM}$, access and use of healthcare services by families with CMC, and healthcare costs and financial burden, compared with standard care. A secondary objective of this study was to identify which telemedicine systems specifically dedicated to CMC are currently used in healthcare.

\section{Materials and methods}

The research questions of this integrative review were as follows: "Does TM in CMC improve the child's health outcomes, the family's ability to engage in self-care, and satisfaction with healthcare services, compared to usual care? Does TM reduce healthcare costs, the financial burden for the family, and the utilization of healthcare services, compared with usual care?".

For these questions, we drafted a protocol based on the population, intervention, comparison, and outcome (PICO) approach and the Preferred Reporting Items for Systematic Reviews and Meta-Analyses (PRISMA) guidelines [43]. To ensure maximum retrieval, two reviewers searched Medline/PubMed, Cumulative Index of Nursing and Allied Health Literature (CINAHL), the Cochrane Library, Web of Science, and Scopus (up to October 2020). See supplementary file 1 for the search query.

Since this was an integrative review, we included both quantitative and qualitative studies [44]. The papers were doubleblinded selected from researchers (FF, OG) according to the following inclusion criteria: (1) pediatric patients $<18$ years of age; (2) CMC as defined by Cohen [3]; (3) studies evaluating telemedicine, telehealth, tele-coordination, and telenursing interventions; and (4) papers in English language. The following exclusion criteria were applied: (1) articles not explicitly describing the application of telemedicine to CMC; (2) studies not describing the association of telemedicine with outcomes such as family and child well-being, child health, the family's ability to implement self-care, adaptive functioning, satisfaction with TM, access and use of healthcare services, healthcare costs, and financial burden; (3) unavailability of full-texts; and (4) reviews and systematic reviews (secondary sources). We extracted data from each paper using the Joanna Briggs Institute Data Extraction Tool for Experimental/Observational Studies and for Qualitative Data [45, 46]. Data were related to the population, the methods, TM interventions, and the outcomes according to the review objectives. Two researchers (FF, 
IE) independently assessed the quality of the retrieved papers using the quality assessment tools of the Joanna Briggs Institute (JBI) (observational, qualitative, and quasi-experimental study designs) and the Cochrane Foundation (Risk of Bias 2.0, for randomized controlled trials). Articles were included if at least 7/10 items on the JBI Check lists were positively evaluated and RCTs if they resulted with a low risk of bias on the ROB 2.0. Disagreements (if any) in the selection, extraction, and quality appraisal processes were resolved by the members of the research team through discussion.

A narrative synthesis for qualitative and quantitative studies was performed. Extracted data were compared item by item; similar data were compared, categorized, and grouped together according to Barnert's classification of CMC outcome domains $[44,47]$. We reported numerical data from quantitative papers as well as point and interval estimates when available.

\section{Results}

We identified 4883 papers across the electronic databases, of which 4714 from Medline/PubMed, 119 from Web of Science (Core Collection), 25 from CINAHL, 23 from Scopus, and 2 from the Cochrane Library. We found a scoping review, from which we retrieved 3 additional papers. At the end of the selection process, we included a total of 18 papers (see the PRISMA Flow Chart in Fig. 1). All the included papers are summarized in Table 1.

\section{Quality of the studies}

All the studies found but one (17 out of 18) showed a low risk of bias or met $\geq 7$ of the quality criteria required by the JBI quality appraisal checklists. A quasi-experimental study [21] was excluded from the qualitative synthesis because of its poor methodology and presentation of results. The details of the quality appraisal are shown in Tables $2-5$, in the electronic supplementary file 2 .

\section{Study designs and sample size}

Of the 17 studies included after quality assessment, 15 were conducted in the USA and 2 in Canada. The included papers were 4 RCTs, 10 quasi-experimental studies, 2 prevalence studies, and one qualitative study. Four studies included the description of the specific outcomes of the TeleFamilies-RCT [48-51]. The sample sizes of the included studies ranged from 11 to $269 \mathrm{CMC}$ or CMC caregivers with a
Fig. 1 Preferred Reporting Items for Systematic Reviews and Meta-Analyses (PRISMA) flow-chart
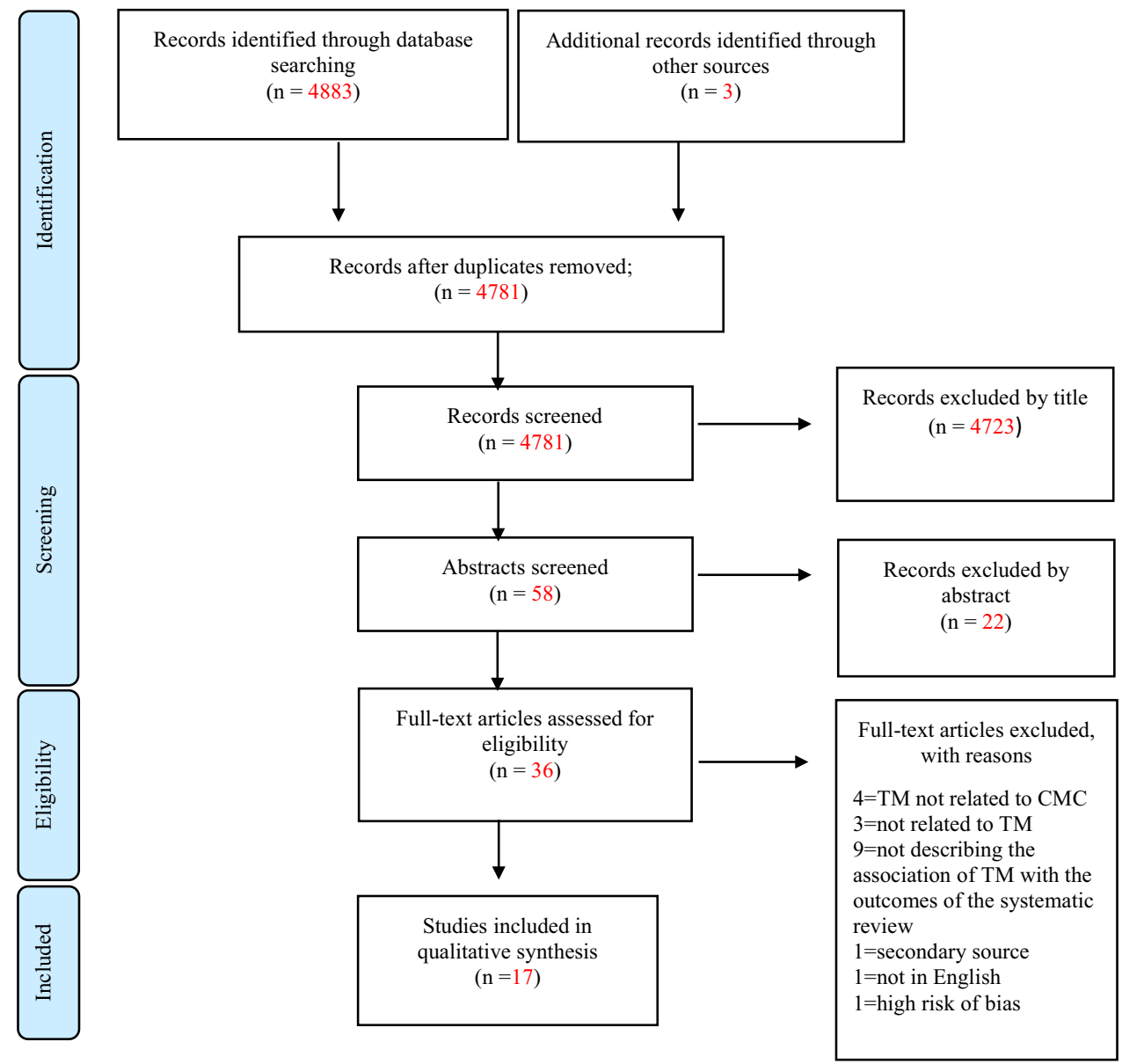


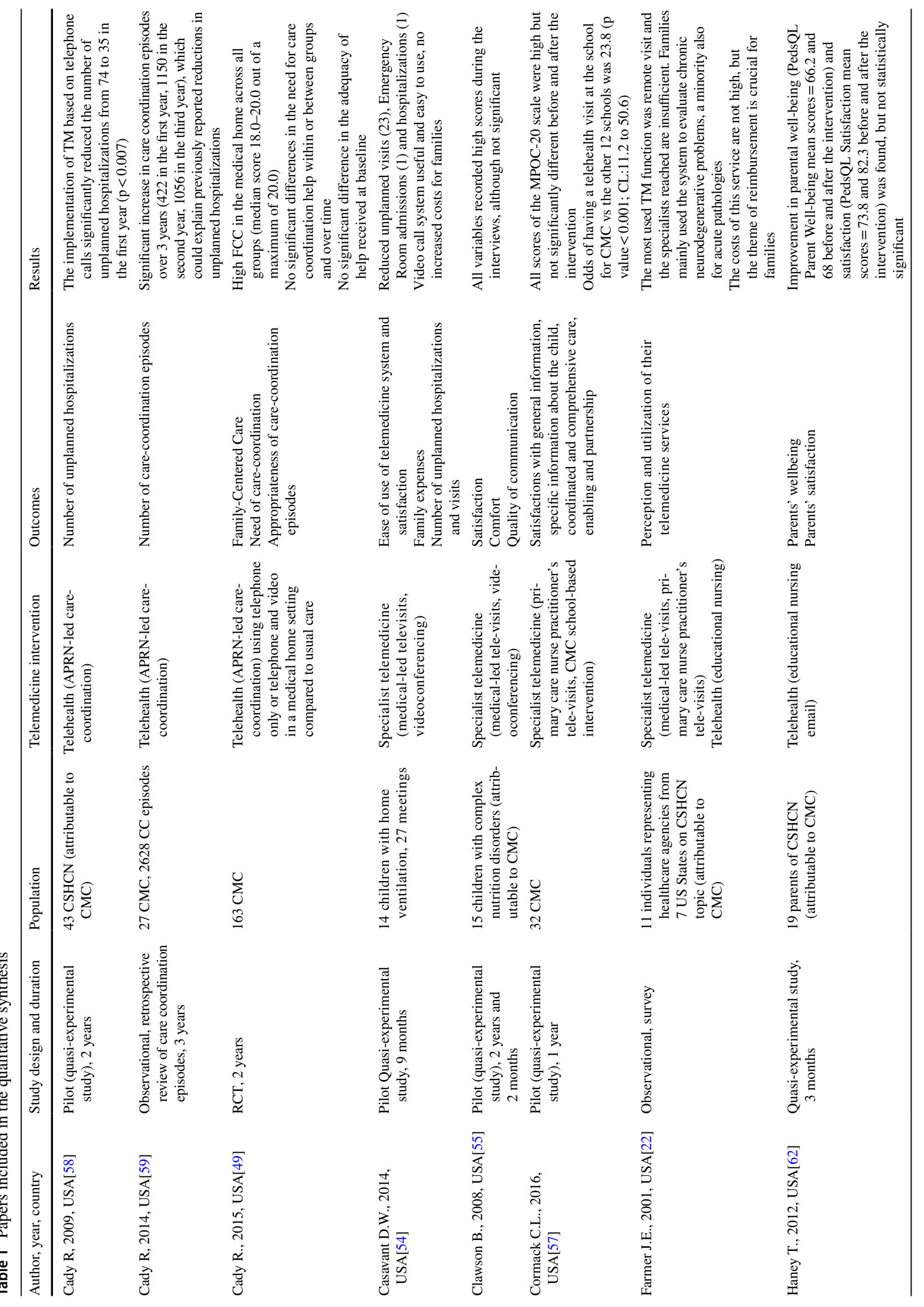




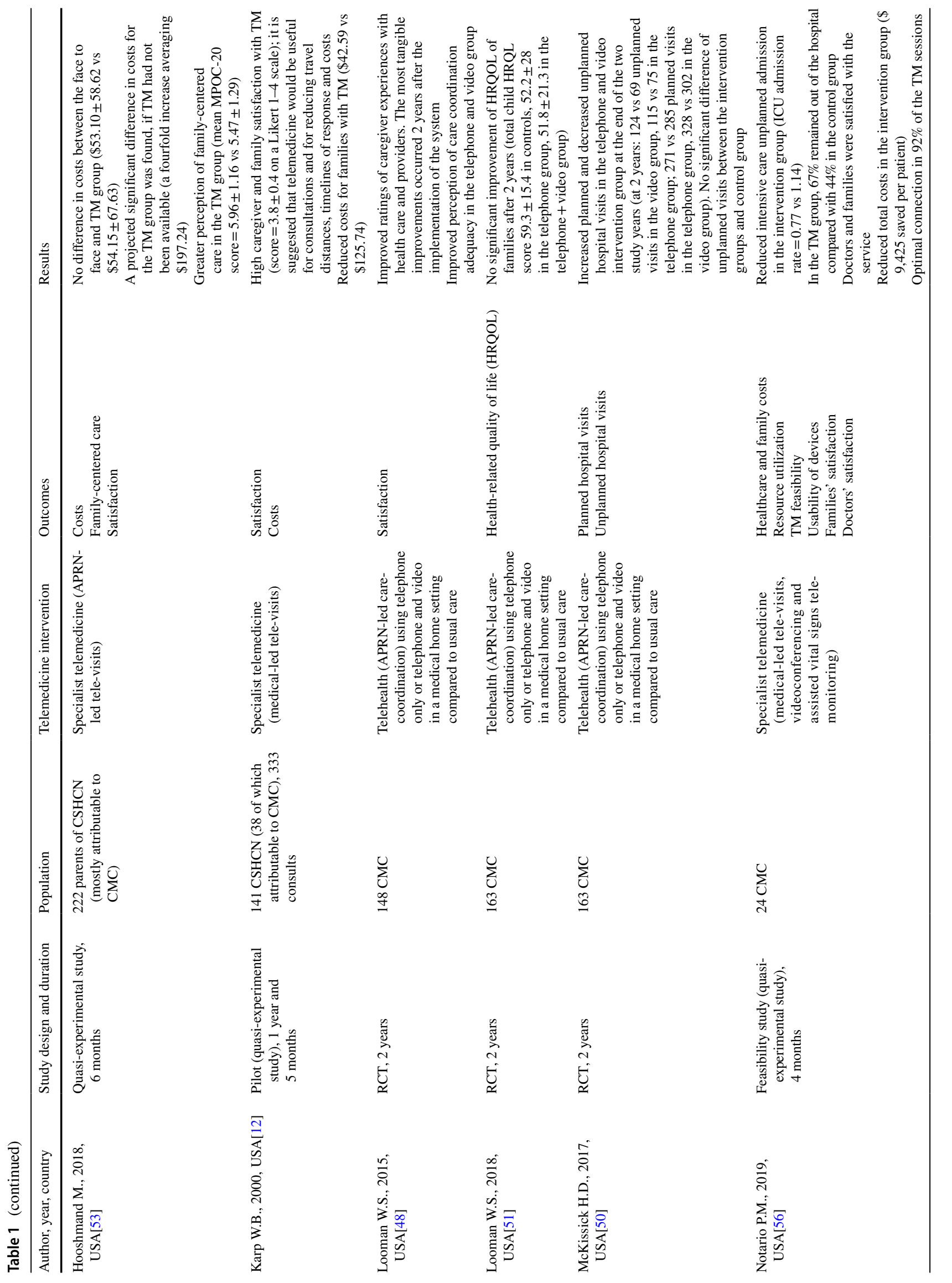


median sample size of 43. In four studies (of which one was TeleFamilies-RCT), the sample size was $>100$ [12, 48-53].

\section{Types of telemedicine systems used}

The studies included in this review included the use of different TM systems, according to the ATA definitions. Eight studies involved Advanced Practice Registered Nursing (APRN) or medical tele-visits [12, 22, 52-57]. Two studies involved the use of a telephone APRN care coordination intervention $[58,59]$. Four studies (TeleFamilies-RCT) involved the use of a telehealth care coordination system run by nurses based on video calls or telephone calls [48-51]. Two studies included tele-monitoring systems [60, 61]. One study involved a telehealth system for sharing educational information and nursing support via e-mail [62].

\section{Outcomes of telemedicine systems}

\section{Use of healthcare services, unplanned hospitalizations, and outpatient visits}

Four studies focused on the association between TM and the use of healthcare services. The use of telemedicine and telehealth systems reduced unplanned hospitalizations but increased scheduled visits. McKissick et al., in the TeleFamilies-RCT, evaluated the effect of care coordination systems in a group of 54 CMC through a TM intervention consisting of telephone or video calls, for 24 months. In this study, a significant increase in planned visits was found in both TM groups, compared with usual care [50]. The video group exhibited the greatest effect with a reduction of unplanned visits equal to $>40 \%$ compared with baseline; no difference between the intervention and control groups was found with regard to the rate of unplanned visits, which were significantly reduced in all study groups compared with baseline until the end of the trial [50].

Cady et al., in a group of $43 \mathrm{CMC}$, found a significant reduction equal to $>50 \%$ of unplanned hospital readmissions (UHR) and unplanned inpatient days from the first year to the second year of a telephone-based care coordination and case management program (first year mean $(\bar{x}$ UHR $1.7 ; \mathrm{SD} \pm 2.5$ vs second year; $\bar{x}$ UHR 0.8 ; $\mathrm{SD} \pm 1.7, \mathrm{p}=0.007)$. Also, during the 5 years of the study, normalized rates of planned inpatient days were constant, while rates of unplanned inpatient days decreased [58]. In another study, Cady et al. found that the use of Care Coordination systems on telephone or video calls through telehealth systems increased the number of episodes of care coordination and nursing consultations in three years [59]. Casavant et al. also found that the use of TM (27 TM encounters with 14 patients) prevented a total of 23 outpatient visits, 3 ED visits, and 1 hospital admission during their study [54]. 


\section{Family well-being: satisfaction with telemedicine systems}

Six studies focused on satisfaction with TM systems [12, 52, 55]. Looman et al., in the TeleFamilies-RCT, found a significant increase on the CG-CAHPS scale in the phone $(\mathrm{P})$ and video $(\mathrm{V})$ intervention groups compared with the control (C) group with regard: communication with healthcare providers, child's health, the relationship with the child's personal physician, and family centered care [48]. During the second year, satisfaction was stable for the TM group but increased in the control group for the latter two items [48]. On the same trial, Cady et al. found that among the Family Centered Care (FCC) items of the CG-CAHPS scale, adequacy of care coordination significantly increased by $10 \%$ in both TM groups during the 2 years of the trial (Perception of adequacy with TM care coordination $=60 \%$ vs control group $<50 \%, p<0.05$ ) [45]. In Hooshmand and Foronda's study, TM also significantly increased the perception of FCC compared with the control group [53]. Cormack et al. used the same scale, but no significant impact on satisfaction was found, possibly because families were already highly satisfied at the baseline and due to the small sample size [57].

\section{Family and child health well-being: quality of life}

In the family quality of life domain, three studies measured family well-being and children's Health Related Quality Of Life (HRQOL) $[48,57,58]$ and a study examined parents' stress through a qualitative analysis [56]. Looman et al. found that TeleFamilies-RCT interventions did not significantly change HRQOL for CMC and families under TM interventions [51]. Haney and Tufts found a non-significant increase in self-reported well-being with an intervention consisting of informative and educational emails sent to family caregivers [62]. Young et al. reported a reduction of stress in a group of parents and/or caregivers of CMC interviewed for a qualitative study [60] and an increase in the quality of life in children and families using a tool specifically designed for the study. No inferential analysis was performed [61].

\section{Healthcare use: costs of medical equipment and services}

One study examined TM healthcare related costs for caring for CMC by measuring the costs of a 4-month TM intervention compared with usual care follow-up. The authors found decreased total healthcare service costs by $\$ 45$, including costs for unplanned Emergency Room and hospital admissions, TM visits, and related equipment (total costs for visits per visit rate: $144,926.20 \$$ for the control group compared to $100,174.55 \$$ for the TM group) [56].

\section{Family quality of life: financial burden}

Two studies reported on the impact of telemedicine on the financial burden for families [53, 54]. Hooshmand and Foronda did not find a significant difference in family costs between the TM intervention group and usual care $(\bar{x}=$ $\$ 53.10 \pm 58.62$, usual care; $\bar{x}=54.15 \pm 67.63$, TM group), as both the TM and the traditional clinic were located in the family's community. However, they calculated a projected significant decrease of 6 months of healthcare-related costs for the family in the TM group associated with reduced travelling to tertiary care centers and reduced waiting time for specialist consultations (Mean family usual care costs: $\$ 197.24 \pm 159.42$ vs mean family costs with TM: $\$ 54.15$ to \pm 67.63 ) [53]. In addition, Casavant et al. through a survey with families of CMC found that total travelling costs to hospitals were reduced with the implementation of TM due to fewer unplanned hospital admissions [54].

\section{Discussion}

The 17 papers included in this integrative review offer a summary of the impact of TM on the use of healthcare services, unplanned and healthcare visits, family satisfaction, family wellbeing, child's HRQOL, and costs. These are the main outcome measures recommended for monitoring the health conditions of CMC at home [47]. We found evidence of the impact of TM on the reduction of unplanned hospitalizations, family satisfaction, and reduction of healthcare and travel costs for CMC.

This review identified the implementation of three main TM systems: telehealth based on nursing care coordination, APRN or medical tele-visits and tele-monitoring. Healthcare teams with APRNs as care coordinators is one of the key characteristics of the Medical Home model, highly recommended for CMC because of improved clinical outcomes and reduced costs [16, 18-21, 49, 52]. Effective care coordination resulted in improved communication among programs based on a partnership between primary care, tertiary care, social services, and patients and their families, including the school system [16, 18, 20, 49, 58]. Tele-visits with APRNs, physicians, and other healthcare professionals have the advantage of reducing the distance between patients' homes and specialists' clinics, reducing absence from work, travel related costs, and improving access to care for vulnerable populations such as CMC [50,52]. Tele-monitoring used simultaneously with tele-visits reduce in-presence healthcare utilization and costs, as well as earlier hospital discharge [53, 57]. Different TM approaches should be further tested, and evidence of iterative process improvement should be provided within different clinical pathways for 
CMC including hybrid solutions according to the patient's illness severity, characteristics, and personal needs.

We found that TM reduced unplanned admissions and hospital visits of CMC patients up to 50\% [50, 58]. This result was similar to other pediatric chronic diseases, such as type I diabetes, where the use of TM systems was found to reduce the onset of complications, such as hyperglycemia or hypoglycemia, reducing unplanned hospitalization [24, 27]. Tele-monitoring and other mobile technologies have the potential to provide healthcare professionals with real time data on the child's health, and to schedule child visits according to data based on actual needs instead of standard follow up times. [20]. Moreover, access to specialized care for $\mathrm{CMC}$ is reduced when families live far from the hospital and in rural low-serviced communities [7]. A high proportion of CMC (25.7\%) accessing the ED require unplanned hospital admissions, increasing preventable healthcare expenditure [7]. Conversely, CMC that receive accessible and timely primary care require fewer ED admissions [7, 63]. APRN TM care coordination improves team communication, access to healthcare services, and timeliness of planned visits.

Family satisfaction with TM systems is high, and predominantly higher in TM care models than in usual care without TM. Family satisfaction is an essential indicator of family perception of care, family involvement, well-being, and care coordination. Currently available instruments used to measure CMC caregivers' satisfaction [47] do not include specific items related to CMC's experience of care. [64] such as time of travel to the hospital and stress related to the complexity of moving CMC's technological devices [55]. Further studies are recommended to investigate validated instruments to measure all the components of CMC caregiver satisfaction.

Child and family quality of life was not found to be significantly affected by TM [51, 61, 62]. Child mental health, health promotion, and family quality of life are poorly studied in CMC despite the relevant role of the family and the burden of caring for these children on their caregivers [47]. A study suggests that in CMC and their families, quality of life is not correlated with healthcare satisfaction, or with the shift from inpatient to outpatient care [65]. However, an association between quality of life, child mental health, and child pain was found [66]. The reasons for this should be further explored with CMC caregivers to maximize the potential of TM to achieve significant patient centered outcomes, such as pain management, child mental health, and caregiver contribution to self-care.

TM was associated with decreased healthcare costs for CMC and decreased financial burden for families [22, 56]. This result is in line with existing literature, including other pediatric populations [67-69]. This is particularly relevant to healthcare for reimbursement policies that include public healthcare expenditure restraint programs $[12,70]$.
Moreover, the association between decreased financial burden in families with CMC and related caregiver satisfaction should be explored [47, 48].

TM has been increasingly used during the COVID-19 pandemic to respond to policies for limiting social contacts in the general population and has proved to be effective in reducing disparities in accessing healthcare services and improved close monitoring and communication with parents of children with complex conditions [71]. During the pandemic, even families of healthy children have been educated to adopt precautions that are well recognized by CMC, and to appreciate some practical advantages of TM. We hope this will speed up the integration of TM into clinical pathways of patients with CMC, and support a general wider use of TM in CMC to increase the flexibility of dedicated health care services, and address the many needs of the families of these children.

\section{Limitations}

Our integrative review has several limitations. Firstly, we included only papers in English and no grey literature was searched. Secondly, TM interventions included in this review were based in Canada and the USA, limiting the generalizability to other countries or different healthcare systems. Moreover, the great diversity of the TM care models reported in the studies does not enable to reach collective conclusions nor the synthesis of research findings into a meta-analysis.

Thirdly, potential risks of bias across studies were found. Except for the Telefamilies RCT, all the other studies presented a risk of selection bias because of convenience sampling, lack of randomization or allocation concealment of the study participants. Families with higher digital literacy were more likely to be included in those studies, potentially excluding socially disadvantaged populations and exacerbating disparities via the "digital divide." Moreover, blinding of participants to the TM intervention and outcome assessment was not possible because of the nature of the intervention, possibly introducing the risk of information bias. On the other hand, attrition bias or selective reporting bias was not found.

Fourth, the relative rarity of CMC over the global pediatric population might be currently limiting the availability of experimental studies evaluating the effect of TM systems in this patient population $[4,5]$. Moreover, most of the included studies had limited sample sizes, therefore reducing the possibility to report significant statistical effects of TM on outcomes for CMC. Despite this limitation, most of the studies documented a significant and relevant magnitude of effect of some outcomes, such as the reduction of unplanned hospital admissions by half. Although rapid cycle testing might be relevant for evaluating rapidly changing TM solutions, 
large multi-centered randomized controlled studies are warranted to investigate the effect of standardized TM models in CMC. Finally, most of the studies included in this review had an observational or quasi experimental design, entailing some degree of uncertainty with regard to the effectiveness of TM systems in improving health outcomes for CMC. As evidence on this topic increases, future reviews should focus on specific domains such as quality of life, mental health, and family self-care skills associated to TM interventions.

\section{Conclusions}

CMC are an extremely fragile patient population that deserves the highest attention to closely monitor their health conditions and prevent complications. Indeed, this population has frequent access to healthcare services compared with other chronic populations. The recent COVID-19 pandemic has stressed the need for healthcare services to push forward the use of TM on a global scale. Still, there are some reports about medical accidents during lockdown due to mobility restrictions and fear of being infected in hospitals especially among patients with chronic conditions [72-74].

This review shows that there is evidence that TM reduces unplanned hospitalizations, healthcare service costs, and financial burden for families, and increases caregiver satisfaction with care. CMC represent a vulnerable population with a high priority for TM programs, particularly at times of increased risk of infections, such as the COVID-19 pandemic. Further studies are needed to confirm the effectiveness of TM systems in improving CMC health and health utilization outcomes, including outcome measures such as mental health, hospital readmissions, caregiver competences, and self-care [47].

Supplementary information The online version contains supplementary material available at https://doi.org/10.1007/s00431-021-04164-2.

\begin{abstract}
Authors' contributions Orsola Gawronski, Maria Luisa Rega, and Federico Ferro conceived the study protocol and planned the work. Federico Ferro and Orsola Gawronski selected the articles. Federico Ferro and Ilaria Erba synthesized data from papers and critically appraised them. Federico Ferro, Alberto Tozzi, and Orsola Gawronski performed the data analysis and interpretation. Federico Ferro, Orsola Gawronski, and Alberto Tozzi drafted the manuscript. Caterina Geremia, Andrea Campana, Gloria Tontini, Immacolata Dall'Oglio, Corrado Cecchetti, and Emanuela Tiozzo critically reviewed the manuscript. Federico Ferro, Ilaria Erba, Corrado Cecchetti, Caterina Geremia, Andrea Campana, Gloria Tontini, Immacolata Dall'Oglio, Emanuela Tiozzo, and Maria Luisa Rega approved the final version.
\end{abstract}

\section{Declarations}

Conflict of interest The authors declare no competing interests.

\section{References}

1. Burns KH, Casey PH, Lyle RE, Bird TM, Fussell JJ, Robbins JM (2010) Increasing prevalence of medically complex children in US hospitals. Pediatrics 126:638-646. https://doi.org/10.1542/ peds.2009-1658

2. Berry JG, Hall M, Neff J, Goodman D, Cohen E, Agrawal R, et al. Children with medical complexity and Medicaid: spending and cost savings. Health Aff (Millwood) 2014;33:2199-206. https:// doi.org/10.1377/hlthaff.2014.0828.

3. Cohen E, Kuo DZ, Agrawal R, Berry JG, Bhagat SKM, Simon $\mathrm{TD}$, et al. Children with medical complexity: an emerging population for clinical and research initiatives. PEDIATRICS 2011;127:529-38. https://doi.org/10.1542/peds.2010-0910.

4. Berry JG, Agrawal RK, Cohen E, Kuo DZ (2013) The landscape of medical care for children with medical complexity

5. Cohen E, Berry JG, Camacho X, Anderson G, Wodchis W, Guttmann A. Patterns and costs of health care use of children with medical complexity. PEDIATRICS 2012;130:e1463-70. https://doi.org/10. 1542/peds.2012-0175.

6. Berry JG, Agrawal R, Kuo DZ, Cohen E, Risko W, Hall M, et al. Characteristics of hospitalizations for patients who use a structured clinical care program for children with medical complexity. J Pediatr 2011;159:284-90. https://doi.org/10.1016/j.jpeds.2011. 02.002 .

7. Coller RJ, Rodean J, Linares DE, Chung PJ, Pulcini C, Hall M, et al. Variation in hospitalization rates following emergency department visits in children with medical complexity. The Journal of Pediatrics 2019;214:113-120.e1. https://doi.org/10.1016/j. jpeds.2019.07.034.

8. Russell CJ, Simon TD. Care of children with medical complexity in the hospital setting. Pediatr Ann 2014;43:e157-162. https://doi. org/10.3928/00904481-20140619-09.

9. Simon TD, Berry J, Feudtner C, Stone BL, Sheng X, Bratton $\mathrm{SL}$, et al. Children with complex chronic conditions in inpatient hospital settings in the United States. Pediatrics 2010;126:64755. https://doi.org/10.1542/peds.2009-3266.

10. Berry JG, Hall M, Hall DE, Kuo DZ, Cohen E, Agrawal R, et al. Inpatient growth and resource use in 28 children's hospitals. JAMA Pediatr 2013;167:170-7. https://doi.org/10.1001/ jamapediatrics.2013.432.

11. Hooshmand M, Yao K. Challenges facing children with special healthcare needs and their families: telemedicine as a bridge to care. Telemed J E Health 2017;23:18-24. https://doi.org/10.1089/ tmj.2016.0055.

12. Karp WB, Grigsby RK, McSwiggan-Hardin M, Pursley-Crotteau $\mathrm{S}$, Adams LN, Bell W, et al. Use of telemedicine for children with special health care needs. Pediatrics 2000;105:843-7.

13. American Telemedicine Association (ATA). Telemedicine, telehealth, and health information technology 2006.

14. Pordes E, Gordon J, Sanders LM, Cohen E. Models of care delivery for children with medical complexity. Pediatrics 2018;141:S212-23. https://doi.org/10.1542/peds.2017-1284F.

15. Antonelli RC, Antonelli DM. Providing a medical home: the cost of care coordination services in a community-based, general pediatric practice. Pediatrics 2004;113:1522-8.

16. Cooley WC. Providing a primary care medical home for children and youth with cerebral palsy. Pediatrics 2004;114:1106-13.

17. Cooley WC, McAllister JW, Sherrieb K, Kuhlthau K. Improved outcomes associated with medical home implementation in pediatric primary care. Pediatrics 2009;124:358-64.

18. Cooley WC, McAllister JW. Building medical homes: improvement strategies in primary care for children with special health care needs. Pediatrics 2004;113:1499-506. 
19. Hoff T, Weller W, DePuccio M. The patient-centered medical home: a review of recent research. Medical Care Research and Review 2012;69:619-44.

20. Perrin JM. Patient-centered medical home for high-risk children with chronic illness. Jama 2014;312:2625-6.Perrin JM. Patientcentered medical home for high-risk children with chronic illness. Jama 2014;312:2625-6.

21. Cady R, Kelly A, Finkelstein S. Home telehealth for children with special health-care needs. J Telemed Telecare 2008;14:1737. https://doi.org/10.1258/jtt.2008.008042.

22. Farmer JE, Muhlenbruck L. Telehealth for children with special health care needs: promoting comprehensive systems of care. Clin Pediatr (Phila) 2001;40:93-8. https://doi.org/10.1177/000992280104000205.

23. Edwards D, Noyes J, Lowes L, Spencer LH, Gregory JW. An ongoing struggle: a mixed-method systematic review of interventions, barriers and facilitators to achieving optimal self-care by children and young people with Type 1 Diabetes in educational settings. BMC PEDIATRICS 2014;14. https://doi.org/10.1186/ 1471-2431-14-228.

24. Sheehy S, Cohen G, R Owen K. Self-management of diabetes in children and young adults using technology and smartphone applications. Current Diabetes Reviews 2014;10:298-301.

25. Shulman RM, O'Gorman CS, Palmert MR. The impact of telemedicine interventions involving routine transmission of blood glucose data with clinician feedback on metabolic control in youth with type 1 diabetes: a systematic review and meta-analysis. International Journal of Pediatric Endocrinology 2010;2010:1-9.

26. Sun C, Malcolm JC, Wong B, Shorr R, Doyle M-A. Improving glycemic control in adults and children with type 1 diabetes with the use of smartphone-based mobile applications: a systematic review. Canadian Journal of Diabetes 2019;43:51-8.

27. Wang Y, Xue H, Huang Y, Huang L, Zhang D. A systematic review of application and effectiveness of mHealth interventions for obesity and diabetes treatment and self-management. Advances in Nutrition 2017;8:449-62.

28. Huang X, Matricardi PM. Allergy and asthma care in the mobile phone era. Clinical Reviews in Allergy \& Immunology 2019;56:161-73.

29. Kew KM, Cates CJ. Remote versus face-to-face check-ups for asthma. Cochrane Database of Systematic Reviews 2016.

30. Morrison D, Wyke S, Agur K, Cameron EJ, Docking RI, MacKenzie $\mathrm{AM}$, et al. Digital asthma self-management interventions: a systematic review. Journal of Medical Internet Research 2014;16:e51.

31. Hammersley ML, Jones RA, Okely AD. Parent-focused childhood and adolescent overweight and obesity eHealth interventions: a systematic review and meta-analysis. Journal of Medical Internet Research 2016;18:e203.

32. Lee J, Piao M, Byun A, Kim J. A systematic review and metaanalysis of intervention for pediatric obesity using mobile technology. 2016.

33. Cox NS, Alison JA, Rasekaba T, Holland AE. Telehealth in cystic fibrosis: a systematic review. J Telemed Telecare 2012;18:728. https://doi.org/10.1258/jtt.2011.110705.

34. Cox NS, Alison JA, Holland AE. Interventions for promoting physical activity in people with cystic fibrosis. Cochrane Database of Systematic Reviews 2013.

35. Goldbeck L, Fidika, A, Herle, M, Quittner A. Psychological interventions for individuals with cystic fibrosis and their families. Cochrane Database of Systematic Reviews 2014. https://doi.org/ 10.1002/14651858.CD003148.pub3.

36. Grist R, Porter J, Stallard P. Mental health mobile apps for preadolescents and adolescents: a systematic review. J Med Internet Res 2017;19:e176. https://doi.org/10.2196/jmir.7332.

37. Boisvert M, Lang R, Andrianopoulos M, Boscardin ML. Telepractice in the assessment and treatment of individuals with autism spectrum disorders: a systematic review. Dev Neurorehabil 2010;13:423-32. https://doi.org/10.3109/17518423.2010.499889.

38. Parsons D, Cordier R, Vaz S, Lee HC. Parent-mediated intervention training delivered remotely for children with autism spectrum disorder living outside of urban areas: systematic review. J Med Internet Res 2017;19:e198. https://doi.org/10.2196/jmir.6651.

39. Sutherland R, Trembath D, Roberts J. Telehealth and autism: a systematic search and review of the literature. Int J Speech Lang Pathol 2018;20:324-36. https://doi.org/10.1080/17549507.2018. 1465123.

40. Fisher E, Law E, Dudeney J, Eccleston C, Palermo TM. Psychological therapies (remotely delivered) for the management of chronic and recurrent pain in children and adolescents. Cochrane Database of Systematic Reviews 2019. https://doi.org/10.1002/ 14651858.CD011118.pub3.

41. Tozzi AE, Carloni E, Gesualdo F, Russo L, Raponi M. Attitude of families of patients with genetic diseases to use m-health technologies. Telemedicine and E-Health 2015;21:86-9. https://doi. org/10.1089/tmj.2014.0080.

42. Wong CA, Ming D, Maslow G, Gifford EJ. Mitigating the impacts of the COVID-19 pandemic response on at-risk children. Pediatrics 2020;146:e20200973. https://doi.org/10.1542/peds. 2020-0973.

43. Liberati A, Altman DG, Tetzlaff J, Mulrow C, Gøtzsche PC, Ioannidis JPA, et al. The PRISMA statement for reporting systematic reviews and meta-analyses of studies that evaluate health care interventions: explanation and elaboration. PLoS Med 2009;6:e1000100. https://doi.org/10.1371/journal.pmed.1000100.

44. Whittemore R, Knafl K. The integrative review: updated methodology. Journal of Advanced Nursing 2005;52:546-53. https://doi. org/10.1111/j.1365-2648.2005.03621.x.

45. Aromataris E, Munn Z. Appendix 2.3: Qualitative data extraction Tool 2020;JBI Manual for Evidence Synthesis. https://doi.org/10. 46658/JBIMES-20-01.

46. Pearson A, Field J, Jordan Z. Appendix 3: Data extraction tools 2006: Evidence-based clinical practice in nursing and health care: assimilating research, experience and expertise. https://doi.org/10. 1002/9781444316544.app3.

47. Barnert ES, Coller RJ, Nelson BB, Thompson LR, Tran J, Chan $\mathrm{V}$, et al. Key population health outcomes for children with medical complexity: a systematic review. Matern Child Health J 2019;23:1167-76. https://doi.org/10.1007/s10995-019-02752-1.

48. Looman WS, Antolick M, Cady RG, Lunos SA, Garwick AE, Finkelstein SM. Effects of a telehealth care coordination intervention on perceptions of health care by caregivers of children with medical complexity: a randomized controlled trial. J Pediatr Health Care 2015;29:352-63. https://doi.org/10.1016/j.pedhc. 2015.01.007.

49. Cady RG, Erickson M, Lunos S, Finkelstein SM, Looman W, Celebreeze M, et al. Meeting the needs of children with medical complexity using a telehealth advanced practice registered nurse care coordination model. Matern Child Health J 2015;19:1497506. https://doi.org/10.1007/s10995-014-1654-1.

50. McKissick HD, Cady RG, Looman WS, Finkelstein SM. The impact of telehealth and care coordination on the number and type of clinical visits for children with medical complexity. J Pediatr Health Care 2017;31:452-8. https://doi.org/10.1016/j.pedhc.2016.11.006.

51. Looman WS, Hullsiek RL, Pryor L, Mathiason MA, Finkelstein SM. Health-related quality of life outcomes of a telehealth care coordination intervention for children with medical complexity: a randomized controlled trial. J Pediatr Health Care 2018;32:6375. https://doi.org/10.1016/j.pedhc.2017.07.007.

52. Robinson SS, Seale DE, Tiernan KM, Berg B. Use of telemedicine to follow special needs children. Telemed J E Health 2003;9:5761. https://doi.org/10.1089/153056203763317657. 
53. Hooshmand M, Foronda C. Comparison of telemedicine to traditional face-to-face care for children with special needs: a quasiexperimental study. Telemed J E Health 2018;24:433-41. https:// doi.org/10.1089/tmj.2017.0116.

54. Casavant DW, McManus ML, Parsons SK, Zurakowski D, Graham RJ. Trial of telemedicine for patients on home ventilator support: feasibility, confidence in clinical management and use in medical decision-making. J Telemed Telecare 2014;20:441-9. https://doi. org/10.1177/1357633X14555620.

55. Clawson B, Selden M, Lacks M, Deaton AV, Hall B, Bach R. Complex pediatric feeding disorders: using teleconferencing technology to improve access to a treatment program. Pediatr Nurs 2008;34:213-6.

56. Notario PM, Gentile E, Amidon M, Angst D, Lefaiver C, Webster $\mathrm{K}$. Home-based telemedicine for children with medical complexity. Telemed J E Health 2019;25:1123-32. https://doi.org/10.1089/ tmj.2018.0186.

57. Cormack CL, Garber K, Cristaldi K, Edlund B, Dodds C, McElligott L. Implementing school based telehealth for children with medical complexity. J Pediatr Rehabil Med 2016;9:237-40. https://doi.org/ 10.3233/PRM-160385.

58. Cady R, Finkelstein S, Kelly A. A telehealth nursing intervention reduces hospitalizations in children with complex health conditions. J Telemed Telecare 2009;15:317-20. https://doi.org/10. 1258/jtt.2009.090105.

59. Cady RG, Kelly AM, Finkelstein SM, Looman WS, Garwick AW. Attributes of advanced practice registered nurse care coordination for children with medical complexity. J Pediatr Health Care 2014;28:305-12. https://doi.org/10.1016/j.pedhc.2013.06.005.

60. Young NL, Barden W, McKeever P, Dick PT. Taking the call-bell home: a qualitative evaluation of Tele-HomeCare for children. Health \& Social Care in the Community 2006;14:231-41. https:// doi.org/10.1111/j.1365-2524.2006.00615.x.

61. Young NL, Bennie J, Barden W, Dick PT. An examination of quality of life of children and parents during their Tele-HomeCare experience. Telemedicine and E-Health 2006;12:663-71. https:// doi.org/10.1089/tmj.2006.12.663.

62. Haney T, Tufts KA. A pilot study using electronic communication in home healthcare: implications on parental well-being and satisfaction caring for medically fragile children. Home Healthcare Nurse 2012;30:216-24. https://doi.org/10.1097/NHH.0b013e31824c28f2.

63. Coller RJ, Nelson BB, Sklansky DJ, Saenz AA, Klitzner TS, Lerner CF, et al. Preventing hospitalizations in children with medical complexity: a systematic review. Pediatrics 2014;134:e162847. https://doi.org/10.1542/peds.2014-1956.

64. Rickwood D, Wallace A, Kennedy V, O'Sullivan S, Telford N, Leicester S. Young people's satisfaction with the online mental health service eheadspace: development and implementation of a service satisfaction measure. JMIR Ment Health 2019;6. https:// doi.org/10.2196/12169.

65. Johaningsmeir SA, Colby H, Krauthoefer M, Simpson P, Conceição SCO, Gordon JB. Impact of caring for children with medical complexity and high resource use on family quality of life. J Pediatr Rehabil Med 2015;8:75-82. https://doi.org/10.3233/PRM-150321.

66. Ellzey A, Valentine KJ, Hagedorn C, Murphy NA. Parent perceptions of quality of life and healthcare satisfaction for children with medical complexity. J Pediatr Rehabil Med 2015;8:97104. https://doi.org/10.3233/PRM-150323.

67. Spaulding R, Cain S, Sonnenschein K. Urban telepsychiatry: uncommon service for a common need. Child Adolesc Psychiatr Clin N Am 2011;20:29-39. https://doi.org/10.1016/j.chc.2010.08. 010 .

68. Burke BL, Hall RW, Care the SOT. Telemedicine: pediatric applications. Pediatrics 2015;136:e293-308. https://doi.org/10.1542/ peds.2015-1517.

69. Dávalos ME, French MT, Burdick AE, Simmons SC. Economic evaluation of telemedicine: review of the literature and research guidelines for benefit-cost analysis. Telemedicine and E-Health 2009;15:933-48. https://doi.org/10.1089/tmj.2009.0067.

70. Doolittle GC, Spaulding AO, Williams AR. The decreasing cost of telemedicine and telehealth. Telemedicine and E-Health 2011;17:671-5. https://doi.org/10.1089/tmj.2011.0033.

71. Menon DU, Belcher HME. COVID-19 pandemic health disparities and pediatric health care - the promise of telehealth. JAMA Pediatr 2021;175:345-6. https://doi.org/10.1001/jamapediatrics. 2020.5097.

72. Peters AL, Garg SK. The Silver Lining to COVID-19: Avoiding diabetic ketoacidosis admissions with telehealth. diabetes technology \& therapeutics 2020;22:449-53. https://doi.org/10.1089/dia. 2020.0187.

73. Portnoy J, Waller M, Elliott T. Telemedicine in the era of COVID19. The Journal of Allergy and Clinical Immunology: In Practice 2020;8:1489-91. https://doi.org/10.1016/j.jaip.2020.03.008.

74. Patel PD, Cobb J, Wright D, Turer RW, Jordan T, Humphrey A, et al. Rapid development of telehealth capabilities within pediatric patient portal infrastructure for COVID-19 care: barriers, solutions, results. J Am Med Inform Assoc n.d. https://doi.org/10. 1093/jamia/ocaa065.

Publisher's Note Springer Nature remains neutral with regard to jurisdictional claims in published maps and institutional affiliations. 


\section{Authors and Affiliations}

\section{Federico Ferro ${ }^{1}$ (D) Alberto Eugenio Tozzi ${ }^{2} \cdot$ Ilaria Erba $^{3} \cdot$ Immacolata Dall'Oglio $^{1}$ (D) $\cdot$ Andrea Campana $^{4}$. Corrado Cecchetti ${ }^{5}$. Caterina Geremia ${ }^{4}$. Maria Luisa Rega ${ }^{6}$. Gloria Tontini ${ }^{4}$. Emanuela Tiozzo ${ }^{1}$. Orsola Gawronski ${ }^{1}$ (D)}

\section{Federico Ferro}

ferrofederico1995@gmail.com

Alberto Eugenio Tozzi

albertoeugenio.tozzi@opbg.net

Ilaria Erba

ila.erba@outlook.it

Immacolata Dall'Oglio

immacolata.dalloglio@opbg.net

Andrea Campana

andrea.campana@opbg.net

Corrado Cecchetti

corrado.cecchetti@opbg.net

Caterina Geremia

caterina.geremia@opbg.net

Maria Luisa Rega

MariaLuisa.Rega@unicatt.it

Gloria Tontini

gloria.tontini@opbg.net

Emanuela Tiozzo

emanuela.tiozzo@opbg.net
1 Medical Directorate, Professional Development, Continuing Education and Research Unit, Bambino Gesù Children's Hospital IRCCS, Rome, Italy

2 Scientific Directorate, Multifactorial Diseases and Complex Chronic Diseases, Bambino Gesù Children's Hospital IRCCS, Rome, Italy

3 Department of Anesthesia and Critical Care, Pediatric Intensive Care Unit, Bambino Gesù Children's Hospital IRCCS, Rome, Italy

4 Department of Emergency, Acceptance and General Pediatrics, Bambino Gesù Children's Hospital IRCCS, Rome, Italy

5 Department of Emergency, Acceptance and General Pediatrics, Pediatric Intensive Care Unit, Bambino Gesù Children's Hospital IRCCS, Rome, Italy

6 Department of Nursing, Sacred Heart Catholic University, Rome, Italy 